\title{
Experimental Analysis of a New Algorithm for Partial Haplotype Completion
}

\author{
Paola Bonizzoni ${ }^{1,3}$, Gianluca Della Vedova ${ }^{2,3}$, \\ Riccardo Dondi ${ }^{1,3}$, and Lorenzo Mariani ${ }^{1,3}$ \\ 1 Dipartimento di Informatica, Sistemistica e Comunicazione \\ 2 Dipartimento di Statistica \\ 3 Univ. Milano-Bicocca, Via Bicocca degli Arcimboldi 8, 20126 Milano(Italy) \\ bonizzoni@disco.unimib.it, riccardo.dondi@unimib.it
}

\begin{abstract}
This paper deals with the computational problem of inferring complete information on haplotypes from haplotypes with missing data. This problem is one of the main issues in haplotyping, as the current DNA sequencing technology often produces haplotypes with missing bases and thus the complete information on haplotypes has to be inferred through computational methods. In this paper we propose a new algorithmic approach to the problem that assumes both the Coalescent and the Minimum Entropy models and we provide an experimental analysis relating it to the previously investigated approaches. In particular, the reconstruction of a perfect phylogeny from haplotypes with missing data is addressed.
\end{abstract}

\section{Introduction}

After the completion of the Human Genome Project, one of the main goals of Molecular Biology is the understanding of the relationship between variants in the human genome sequence and many common diseases influenced by multiple genetic and environmental factors. The main genetic differences among individuals concern about ten million single DNA sequence sites, referred to as Single Nucleotide Polymorphisms (SNPs), in which usually two bases are observed across the population. Therefore, when studying differences among human DNA sequences, only the bases at SNP sites are considered and other bases can be ignored. The sequence of the bases that an individual presents in all or some of his SNP sites is called a haplotype.

Recent studies 4 have shown that the human DNA sequence can be subdivided into blocks that have been transmitted through evolution without recombination, and that within each block a few (2-5) distinct common haplotypes that cover about $70-90 \%$ of the total observed haplotypes. The National Institutes of Health has launched the Haplotype Map Project [9], whose goal is the identification of the haplotype blocks and the common haplotypes in the genome of different human populations. This project has encouraged the development of computational methods to address several issues and indeed haplotyping [1] is a research topic which poses new challenging questions to computer scientists. 
A main issue in haplotyping is the inference of complete haplotypes from haplotypes with missing data as the current DNA sequencing technology often produces haplotypes with missing bases at some positions. Combinatorial methods based on inference rules represent a common approach to face this problem [7. These methods usually use inference rules based on assumptions on evolutionary model for human haplotypes or statistical analysis of haplotypes frequency. Two of the main patterns adopted for this problem are the coalescent model (shortly CM) and the minimum entropy model (shortly MEM). The coalescent model, proposed in [5] describes the evolutionary relationships between haplotypes, assuming that no recombination occurs between haplotypes and that at each SNP site a mutation can happen only once during evolution. The minimum entropy model, proposed in [6], assumes that the frequency distribution of the haplotypes within the population has a small entropy.

Some existing algorithms assume either CM or MEM. Algorithms assuming both of them, require some strict hypotheses to be satisfied (for example, knowing the phylogenetic tree of the haplotypes) [7. In this paper we propose a new algorithm that assumes both CM and MEM, requiring only one complete haplotype to be known.

Our algorithm consists of two phases. First, it produces a completion of the partial haplotypes that fits $\mathrm{CM}$, implementing a variant of the "Naïve Algorithm A" of [10]. Then, it executes a local optimization heuristic. In particular, two different procedures have been designed, implemented and tested for this second phase: a greedy improvement procedure and a Kernighan-Lin heuristic style.

Various experiments have been performed in order to study the performance of the algorithm. Experimental results show that the amount of data incorrectly completed is a very low percentage with respect to the amount of total missing data, suggesting that both the greedy improvement heuristic and the Kernighan-Lin heuristic are good approaches for the local optimization of the solution, especially when they are combined. The performance of an algorithm that adopts either the two heuristics has been compared with those of other effective softwares [7, 6, 3]. The results of the comparison are encouraging, showing the effectiveness of the new approach proposed.

\section{The Partial Haplotype Completion Problem}

In diploid organisms like humans only two different bases are observed in each SNP site, leading to a natural $\{0,1\}$-representation. Actually, current sequencing technology often produces DNA sequences with some missing bases at some position. Therefore, we must adopt a $\{0,1, ?\}$-representation to denote the values assumed by each SNP site, where '?' represents the lack of data.

Let $S=\left\{s_{1}, s_{2}, \ldots, s_{n}\right\}$ denote a set of $n$ individuals and let $C=\left\{c_{1}, c_{2}, \ldots, c_{m}\right\}$ denote a set of $m$ SNP sites observed in a specified block of the human genome. Then, a complete haplotype over the SNP sites in $C$ is a $\{0,1\}^{m}$-vector. A partial haplotype over the SNP sites in $C$ is a $\{0,1, ?\}^{m}$-vector. A partial haplotype $p h$ is said to be compatible with a complete haplotype $c h$ if and only if $p h[i] \neq$ '?' 
implies that $\operatorname{ch}[i]=p h[i]$, for $i=1,2, \ldots, m$. Given a matrix $K$, we denote with $K(i)$ the i-th row of $K$ and with $K_{i j}$ the entry corresponding to the i-th row and the j-th column of $K$. The set of partial haplotypes associated to the set $S$ of individuals is represented by a $\{0,1, ?\}$-matrix $\mathcal{A}$, called partial haplotypes matrix, where each row $\mathcal{A}(i)$ represents a partial haplotype for individual $s_{i} \in S$. A $\{0,1\}$-matrix $\mathcal{M}$ is a complete haplotypes matrix if it is the completion of $\mathcal{A}$ such that each row $\mathcal{A}(i)$ is compatible with $\mathcal{M}(i)$, for $i=1, \ldots, n$.

In the partial haplotype inference we assume two models commonly adopted when dealing with this problem, the coalescent model and the minimum entropy model, described below.

The coalescent model. Recent studies 4 have shown that the human genome can be partitioned into blocks that have been transmitted without showing evidence for recombination. Moreover, recurrent mutations at the same SNP site are rare events in the evolutionary history. Thus, we can assume that the common haplotypes observed in the human population within a genome block fits the CM[5]. The CM describes the evolutionary relations between haplotypes assuming that: (i) there is no recombination between haplotypes and (ii) at each SNP site a mutation can happen only once. By the assumptions of the model, a haplotype with a mutation at a SNP site is a descendant of the ancestor haplotype $h_{i}$ in which the mutation first occurred, while a haplotype without the mutation cannot be a descendant of $h_{i}$. This implies that the evolutionary history of the collected haplotypes is described by a phylogenetic tree known as perfect phylogeny.

The problem of finding a completion of a partial haplotypes matrix so that the haplotypes in the complete matrix fit the CM is NP-hard 11. Nevertheless, it becomes tractable when at least one complete haplotype is known [10]: in this case it is possible to change the $\{0,1\}$-representation of the bases at each SNP site in such a way that the known complete haplotype is represented by the all-zero vector. Indeed, in terms of evolutionary tree, this corresponds to fix the known all-zero haplotype as the root of the perfect phylogeny, which becomes the so-called directed perfect phylogeny. In what follows we assume that the allzero vector always belong to the perfect phylogeny, i.e. we adopt the directed or rooted model.

Let $\mathcal{A}$ be a partial haplotypes matrix then, given two columns $j_{1}, j_{2}$ with $1 \leq j_{1}<j_{2} \leq m$, the set of the valid pairs for $j_{1}$ and $j_{2}$ is the set $\operatorname{VP}\left(j_{1}, j_{2}\right) \subseteq$ $\{(0,0),(0,1),(1,0),(1,1)\}$ such that $(x, y) \in V P\left(j_{1}, j_{2}\right)$ if and only if there exists the pair $a_{i j_{1}}, a_{i j_{2}}$ of values in a row $i$, with $1 \leq i \leq n$, such that $\left(a_{i j_{1}}, a_{i j_{2}}\right)=$ $(x, y)$.

A characterization of the matrices fitting the CM has been given in [7]. Let $\mathcal{M}$ be a complete haplotype matrix, then $\mathcal{M}$ fits the $\mathrm{CM}$ if and only if for every pair of columns $j_{1}, j_{2} \in\{1,2, \ldots, m\}$ the set of the valid pairs $\operatorname{VP}\left(j_{1}, j_{2}\right)$ is such that $\{(0,1),(1,0),(1,1)\} \nsubseteq V P\left(j_{1}, j_{2}\right)$.

The minimum entropy model. The minimum entropy model is mainly based on a parsimony principle suggested by the fact that in many real-life situations a frequency distribution tends to have a very low entropy value [7, 6]. 
Assume that a collection of $n$ partial haplotypes $P H=\left\{p h_{1}, p h_{2}, \ldots, p h_{n}\right\}$ of length $m$ is given: these are represented by a partial halpotypes matrix $\mathcal{A}$. Let $\alpha: P H \rightarrow\{0,1\}^{m}$ be the completion function that assigns each partial haplotype $p h \in P H$ to a complete compatible haplotype $\alpha(p h) \in\{0,1\}^{m}$. Thus, let $H=$ $\{\alpha(p h): p h \in P H\}=\left\{h_{1}, h_{2}, \ldots, h_{k}\right\} \subseteq\{0,1\}^{m}$ be the set of the distinct complete haplotypes assigned to the partial haplotypes in $P H$. Let $f_{i}$ be the number of partial haplotypes in $P H$ that are assigned to the complete haplotype $h_{i} \in H: f_{i}=\left|\left\{p h \in P H: \alpha(p h)=h_{i}\right\}\right|$ for every $i=1,2, \ldots, k$, with $f_{1}+f_{2}+$ $\ldots+f_{k}=n$. Thus $\left(f_{1} / n, f_{2} / n, \ldots, f_{k} / n\right)=\left(p_{1}, p_{2}, \ldots, p_{k}\right)$ is the distribution of the relative frequencies of the complete haplotypes assigned to the partial haplotypes in $P H$.

Observe that the haplotypes $\alpha\left(p h_{1}\right), \alpha\left(p h_{2}\right), \ldots, \alpha\left(p h_{n}\right)$ give the rows of a complete haplotypes matrix $\mathcal{M}$ of size $n \times m$ that is the completion of the matrix $\mathcal{A}$. Then the entropy of $\mathcal{M}$ is the entropy of the relative frequency distribution $\left(p_{1}, p_{2}, \ldots, p_{k}\right)$, defined as $\operatorname{ENT}(\mathcal{M})=\operatorname{ENT}\left(p_{1}, p_{2}, \ldots, p_{k}\right)=\sum_{i=1}^{k}\left(-p_{i} \log _{2} p_{i}\right)$.

The MEM assumes that the most likely valid completion of the partial haplotypes is the one that minimizes the entropy value $E N T(\mathcal{M})$; it is proved that finding such a completion is an APX-hard problem [6].

\section{An Algorithm for Partial Haplotype Completion}

We propose a new algorithm for the completion of a collection of partial haplotypes, represented as a $\{0,1, ?\}$-matrix, assuming that both the $\mathrm{CM}$ and the MEM hold. More precisely, the use of the two models lead to search for the partial haplotypes completion that determines the minimum entropy frequency distribution, within the set of the completions that fit the CM. Formally, each instance of the Partial Haplotype Completion Problem is a partial haplotype matrix $\mathcal{A}$ of size $n \times m$, and the goal is to find (if it exists) a matrix $\mathcal{M}$ of size $n \times m$ that is a completion of $\mathcal{A}$ such that $\mathcal{M}$ fits the $\mathrm{CM}$ and $\operatorname{ENT}(\mathcal{M})$ is minimum.

We do not know whether there is a polynomial-time algorithm to find such a completion matrix $\mathcal{M}$, similarly to the case in which the evolutionary history of the complete haplotypes is known [7. Thus, we propose a heuristic algorithm for the problem composed of two phases: first we compute an initial completion that fits the CM, if it is possible, then such completion is modified in order to find a completion that still fits the CM and that determines a smaller entropy.

For the first phase, we design a variant of the "Naïve Algorithm A" proposed in 10. for the incomplete directed perfect phylogeny reconstruction and that we denoted by $(p p)$. When this algorithm is invoked on a $\{0,1, ?\}$-matrix of size $n \times m$, its computational complexity is $O(h n m)$, where $h \leq \min \{m, n\}$.

The results produced in the first phase are refined in the second phase of the algorithm. In particular we use two local optimization heuristic procedures: a greedy heuristic (gh) and a Kernighan-Lin style heuristic (klh), described below. In Section 4 we compare the performance of the two procedures when executed separately or combined. 
The greedy heuristic. The greedy heuristic procedure (gh) is inspired by the greedy algorithm proposed in [6, whose goal is finding a minimum entropy set of complete haplotypes compatible with a given collection of partial haplotypes. Given a partial haplotype matrix $\mathcal{A}$ and a completion matrix $\mathcal{M}$ of $\mathcal{A}$ produced by (pp), the procedure identifies the distinct complete haplotypes of $\mathcal{M}$ and calculates their frequencies. Then, it simply takes each distinct complete haplotype $h$ of $\mathcal{M}$ starting from the most frequent one and ending with the least frequent one, and assigns it to the not-yet-assigned compatible partial haplotypes of $\mathcal{A}$, defining a new completion matrix $\mathcal{M}^{\prime}$. The goal is to concentrate the maximum possible number of haplotypes into large sets, reducing the entropy of their frequency distribution.

The procedure runs in $O\left(n^{2} m\right)$ time, since it takes $O\left(n^{2} m\right)$ time to detect the distinct complete haplotypes of $\mathcal{M}$ and their frequencies, $O(n)$ time to rearrange them in growing order of frequency (with counting sort algorithm) and $O\left(n^{2} m\right)$ time to assign these complete haplotypes to partial haplotypes of $\mathcal{A}$. In some cases, a great set of identical haplotypes can be obtained only considering complete haplotypes different from the ones obtained by applying (pp) and for this goal we have designed a Kernighan-Lin heuristic (klh) 8 .

The Kernighan-Lin heuristic. The Kernighan-Lin style heuristic we propose applies a local improvement of the solution based on the iterative inversion (from ' 0 ' to ' 1 ', or from ' 1 ' to ' 0 ') of the value of an entry $m_{i j}$ of the completion matrix $\mathcal{M}$ that corresponds to a missing data in $\mathcal{A}$, that is $a_{i j}=$ '?'. Note that this inversion can be applied only if the resulting matrix $\mathcal{M}^{\prime}$ still fits the CM. Thus, before operating the inversion we must check, with respect to the Valid Pairs Theorem, the valid pairs of each pair of columns of the matrix $\mathcal{M}^{\prime}$ that we would obtain performing the inversion. This operation takes $\Theta\left(\mathrm{nm}^{2}\right)$.

Moreover, we must design an efficient function that, given a completion matrix $\mathcal{M}$ of matrix $\mathcal{A}$, the complete haplotypes of $\mathcal{M}$ and their frequency distribution, calculates the entropy of the frequency distribution that we would obtain inverting a specific entry $m_{i j}$ (with $a_{i j}={ }^{\prime} ?$ '). Identifying the complete haplotypes of $\mathcal{M}$ and determining their frequency distribution takes $O\left(n^{2} m\right)$ time. Calculating the new entropy of the frequency distribution, instead, takes $O(n m)$ time, since comparing two haplotypes of length $m$ takes $O(m)$ time and such a comparison is performed for all the complete haplotypes, that are at most $n$.

The total number of iterations cannot be determined a priori, since it depends on the random selections at execution time, however the core of the algorithm runs in $O\left(n^{2} m^{2}\right)$ time.

\section{$4 \quad$ Results}

Our algorithm has been tested on input data coming from the common haplotypes found in the blocks $1-7$ and 10-11 of a region of chromosome 5p31, as defined by Daly et al. in 2. Starting with this data, we have constructed 9 complete haplotypes $\{0,1\}$-matrices (matrices $\mathcal{C}$ ), composed of 86-97 rows and 5-31 columns and then, adding missing data to them, almost 40 partial haplotypes 
Algorithm 1: Kernighan Lin Heuristic (klh)

Data: a $\{0,1, ?\}$-matrix $\mathcal{A}$ of size $n \times m$, an integer parameter max_iter; a real number parameter base

compute the distinct complete haplotypes of $\mathcal{M}$, and the frequency distribution;

$\mathcal{B}=\mathcal{M} ; \quad / / \mathcal{B}=$ so-far best found completion

min_entropy $=$ entropy of $\mathcal{M}$;

while (number of iterations performed without improvement $<$ max_iter) do

foreach '?'-entry $a_{i j}$ of $\mathcal{A}$ do

if the inversion of $m_{i j}$ in $\mathcal{M}$ is admissible then

new_entropy $=$ entropy of $\mathcal{M}$ with $m_{i j}$ inverted; $p\left(a_{i j}\right)=b_{a s e^{-n e w \_e n t r o p y}}$

if new_entropy < min_entropy then

$\mathcal{B}=\mathcal{M} ; \quad / /$ an improvement has been obtained

invert $b_{i j}$

min_entropy $=$ new_entropy;

end

else $p\left(a_{i j}\right)=0$;

with probability $p\left(a_{i j}\right)$ for each '?'-entry $a_{i j}$ of $\mathcal{A}$, select a '?'-entry $a_{\hat{i} \hat{j}}$;

invert $m_{\hat{i} \hat{j}}$ in $\mathcal{M}$;

update the number of occurrences of all valid pairs for each column pair of $\mathcal{M}$

update the distinct complete haplotypes of $\mathcal{M}$, and the frequency distribu-

end tion;

Result: $\mathcal{B}$

$\{0,1, ?\}$-matrices (matrices $\mathcal{A}$ ) whose rate of unknown data varied from $1 \%$ to $50 \%$ have been obtained.

The completion of each of these $\{0,1, ?\}$-matrices (matrices $\mathcal{M}$ ) has been computed by adopting four different approaches: executing the single procedure (pp); executing (pp) and (gh); executing (pp) and (klh); executing (pp), followed by the execution of (gh) and then (klh) (we denote this algorithm as $(\mathrm{pp})+(\mathrm{gh})+(\mathrm{klh}))$. For the executions of $(\mathrm{klh})$, different values have been assigned to its two parameters: the base of the exponential used to calculate the inversion probability (from 10 to 100) and the maximum number of iterations without improvements (from 5 to 25 ).

For each completion matrix $\mathcal{M}$ obtained for an incomplete haplotype matrix we have evaluated the error rate, that is the number of entries of $\mathcal{M}$ that are different from the corresponding entries of $\mathcal{C}$, divided by the total number of '?' entries of $\mathcal{A}$, and the entropy difference, that is the difference between the entropy values of matrices $\mathcal{C}$ and $\mathcal{M}$ and are reported in Table 1.

Observe that Table 1 shows that some incomplete matrices admit completion matrices with an entropy lower than that of the complete matrices from which they have been generated; this fact especially happens when the number of sampled individuals is small. The Table also suggests that the greedy heuristic 
Table 1. Comparing the entropy values of matrices $\mathcal{C}$ and $\mathcal{M}$

\begin{tabular}{|l|c|c|c|c|c|c|c|c|}
\hline & \multicolumn{4}{|c|}{ Entropy differences } & \multicolumn{4}{c|}{ Error rates } \\
Approach & Mean & Min & Max & Std.Dev & Mean & Min & Max & Std.Dev \\
\hline (pp) & 0.078 & -0.210 & 0.414 & 0.160 & $4.20 \%$ & $0.00 \%$ & $13.63 \%$ & 0.0420 \\
(pp+klh) & 0.043 & -0.210 & 0.414 & 0.137 & $3.42 \%$ & $0.00 \%$ & $12.81 \%$ & 0.0400 \\
(pp+gh) & -0.001 & -0.210 & 0.414 & 0.108 & $2.64 \%$ & $0.00 \%$ & $11.30 \%$ & 0.0350 \\
(pp+gh+klh) & -0.006 & -0.210 & 0.414 & 0.106 & $2.51 \%$ & $0.00 \%$ & $11.30 \%$ & 0.0339 \\
\hline
\end{tabular}

produces better results than (klh), but the best performance is obtained by combining the two algorithms. The recorded execution times have been always under one second (on a $1.60 \mathrm{GHz}$ processor).

In order to evaluate the scalability of the algorithm, we have tested it on 500 artificial $\{0,1, ?\}$-matrices composed by 50-250 rows and 50-250 columns. Although the average entropy differences and the average error rates show a behaviour that is similar to the one described above, this experiment shows that (klh) strongly affects the execution time of the algorithm (determining a running time up to 600 seconds), when the number of sampled SNP sites is great or when there is an high rate of missing data. Thus, in cases in which the input instance has a very great number of SNP sites or an high rate of unknown data, the use of the $(\mathrm{pp}+\mathrm{gh})$ approach seems to be the best solution.

For the above reasons we have tested the $(\mathrm{pp}+\mathrm{gh})$ approach on 20 artificial $\{0,1, ?\}$-matrices of size $10,000 \times 1,000$, varying the rate of unknown data from $1 \%$ to $40 \%$. We have obtained an error rate with range from $0 \%$ to $0.000005 \%$ and an error percentage up to $0.000002 \%$. The error rates are very small, since we have already observed that the error rate tends to quickly decrease as the number of SNP sites grows. Furthermore, the algorithm has required an average execution time of 54 seconds (ranging from 17 to 113 seconds) on a 1.60 $\mathrm{GHz}$ processor; this shows that the $(\mathrm{pp}+\mathrm{gh})$ approach scales very well to large data sets.

\section{Discussion}

In this section we remark the differences between $(\mathrm{pp}+\mathrm{gh}+\mathrm{klh})$ algorithm and other algorithms proposed for the partial haplotype completion problem. First of all we discuss the differences in terms of models and hypothesis assumed. Two algorithms proposed in [7] assume both CM and MEM, but they both require that the perfect phylogeny of the complete haplotypes is known, while our algorithm only requires the knowledge of one complete haplotype.

The algorithm proposed in [6] assumes only the MEM; it is based on a greedy approach, and runs in exponential time when the complete haplotypes are not known and the number of SNP sites is not logarithmic with respect to the number of individuals. Two algorithms proposed in [7, assume only the CM and require the input data to meet the rich data hypothesis, according to which each pair columns contains three valid pairs. Under this hypothesis the problem 
of completing the partial haplotypes of $\mathcal{A}$ assuming the coalescent model is polynomial [6]. Next we focus on the comparison between the $(\mathrm{pp}+\mathrm{gh}+\mathrm{klh})$ algorithm with other published algorithms, based on experimental data.

On real data, when the rate of missing data is no more than $20 \%$, our algorithm has given an average error rate of $0.85 \%$ (with range from $0.00 \%$ to $6.52 \%$ ) and an average percentage of errors of $0.17 \%$ (with range from $0.00 \%$ to $1.30 \%$ ) with respect to the total number of entries.

We compare these experimental results with those of two algorithms of 7 , 3. Both these algorithms are executed on all the haplotypes found within a block and not only on the common ones, as our algorithm does. Moreover the algorithm proposed in 3 considers as input a collection of genotypes rather than haplotypes, thus it even includes a procedure for the inference of haplotypes from genotypes. Hence, the performance results that we report do not allow a detailed comparison, but they still give a new and interesting point of view.

The greedy algorithm proposed in [7] has been executed on real-data matrices composed by $90-129$ rows and 10-31 columns, with missing data rate varying from $0.5 \%$ to $10 \%$; it has given average error rates between $2.8 \%$ and $8.1 \%$.

The maximum-likelihood-based algorithm proposed in 3 has been executed on real-data matrices composed by 129 rows and 5-31 columns, with a missing data rate of $10.03 \%$; it has given a percentage of errors of $0.23 \%$ with respect to the total number of entries, with a running time of a few seconds.

\section{References}

1. P. Bonizzoni, G. Della Vedova, R. Dondi, and J. Li. The haplotyping problem: a view of computational models and solutions. Journal of Computer and Science Technology, 18:675-688, 2003.

2. M. J. Daly, J. D. Rioux, S. F. Schaffner, and et al. High-resolution haplotype structure in the human genome. Nat. Genet., 29(2):229-232, 2001.

3. E. Eskin, E. Halperin, and R. M. Karp. Large scale reconstruction of haplotypes from genotype data. In Proceedings of the 7th RECOMB, pages 104-113, 2003.

4. S. B. Gabriel, S. F. Schaffner, and et al. The structure of haplotype blocks in the human genome. Science, 296:2225-2229, 2002.

5. D. Gusfield. Haplotyping as perfect phylogeny: Conceptual framework and efficient solutions. In Proceedings of the 6th RECOMB, pages 166-175, 2002.

6. E. Halperin and R. M. Karp. The minimum-entropy set cover problem. In Proceedings of the 31st ICALP, pages 733-744, 2004.

7. E. Halperin and R. M. Karp. Perfect phylogeny and haplotype assignment. In Proceedings of the 8th RECOMB, 2004.

8. K. Helsgaun. An effective implementation of the Lin-Kernighan traveling salesman heuristic. European Journal of Operational Research, 126:106-130, 2000.

9. National Institutes of Health. Large-scale genotyping for the haplotype map of the human genome. RFA (Request For Applications) HG-02-005, 2002.

10. I. Pe'er, T. Pupko, R. Shamir, and R. Sharan. Incomplete directed perfect phylogeny. SIAM Journal on Computing, 33(3):590-607, 2004.

11. M. Steel. The complexity of reconstructing trees from qualitative characters and subtrees. Journal of Classification, 9:91-116, 1992. 